Territorios 36 / Bogotá, 2017, pp. 69-86

ISSN: 0123-8418

ISSNe: $2215-7484$

Ciudades y conflictos en América Latina: ayer, hoy y mañana (I)

\title{
La incorporación de lo indígena en el espacio urbano paceño a principios del siglo XX
}

The Incorporation of the Indigenous in La Paz's Urban Space in the Early $20^{\text {th }}$ Century

A incorporação do indigena no espaço urbano pacenho

a princípios do século $X X$

Nadia Guevara Ordóñez ${ }^{*}$

Recibido: 19 de abril de 2016

Aprobado: 21 de octubre de 2016

Doi: http://dx.doi.org/10.12804/revistas.urosario.edu.co/territorios/a.5093

Para citar este artículo:

Guevara Ordoñez, N. (2017). La incorporación de lo indígena en el espacio urbano paceño a principios del siglo XX. Territorios, (36), 69-86. Doi: http://dx.doi.org/10.12804/revistas.urosario.edu.co/territorios/a.5093

\begin{abstract}
* Institut für Kulturwissenschaft Humboldt Universität zu Berlin. Becaria de investigación del Instituto Francés de Estudios Andinos IFEA-UMIFRE 17 MAEDI/CNRS USR 3337 América Latina. Correo electrónico: guevara. ordonez@yahoo.es.ORCID: http://orcid.org/00000003-3724-8862
\end{abstract}


Palabras clave

Espacio urbano, modernidad, indigenas, apertura urbana, vestimenta, espacio público.

Keywords

urban space, modernity, indigenous peoples, opening of urban spaces, clothing, public space.

Palavras-chave

Espaço urbano, modernidade, indigenas, abertura urbana, vestimenta, espaço público.

territarias 36 70

\section{RESUMEN}

Se realiza un análisis de las implicaciones de la entrada de la "modernidad" en el espacio urbano paceño y su relación con la configuración de las identidades y relatos sobre lo indígena a principios del siglo XX. El análisis se centra en la interconexión entre el discurso sobre modernidad, civilización y progreso con las operaciones físicas urbanas. Los aspectos a ser considerados parten del análisis de la operación de apertura del espacio paceño y su influencia sobre la especialización de los espacios públicos, la puesta en escena de los cuerpos en estos espacios y la regulación y prácticas de jerarquización a partir de la incorporación de nuevos medios de trasporte y la construcción de vías de comunicación.

\section{ABSTRACT}

This paper is an analysis of the implications of "modernity" and its entrance into La Paz's urban space together with its relationship with the configuration of indigenous identities and stories in the early twentieth century. It focuses on the interconnection between the discourses of modernity, civilization and progress with physical, urban operations. Aspects to be considered are based on an analysis of the opening of La Paz spaces and its influence on the specialization of public spaces, the staging of bodies in these spaces and the hierarchy, regulation and practices of the same, starting from the incorporation of new means of transport and the construction of roads.

\section{RESUMO}

Realiza-se uma análise das implicações da entrada da "modernidade" no espaço urbano pacenho e a sua relação com a configuração das entidades e relatos sobre o indígena a princípios do século XX. A análise centra-se na interconexão entre o discurso sobre modernidade, civilização e progresso com as operações físicas urbanas. Os aspetos a ser considerados partem da análise da operação de apertura do espaço pacenho e a sua influência sobre a especialização dos espaços públicos, a posta em cena dos corpos nestes espaços, e a regulação e práticas de hierarquização a partir da incorporação de novos meios de transporte e a construção de vias de comunicação. 
"Las calles de las ciudades son como las páginas de un libro donde se anotan los hechos salientes del pasado. Por los nombres o fechas con que se designan, conocemos las historias de las tradiciones de los pueblos. En las calles y plazas tuvieron lugar las luchas por la religión y la nacionalidad. Muchas de las batalles ideológicas se decidieron en ellas, donde expresaron las multitudes sus demandas sociales”.

Alfredo Sanjinés, 1948

\section{Introducción}

Las ciudades latinoamericanas a fines del siglo XIX presentaban aún varias de las características heredadas de la Colonia. El ingreso de estos países a la economía mundial, al igual que el incremento de su población, fue cambiando las condiciones materiales de las mismas, obligando a la apertura del espacio urbano, es decir, la expansión de la mancha urbana y la construcción de barrios y vías de transporte para comunicarlos. En el caso de varias ciudades andinas, como en el de la ciudad de La Paz, fueron además otros hechos los que jugaron en la reconfiguración del espacio urbano. La Guerra Federal de 1898 y el traslado de la sede de Gobierno a esta ciudad fue el factor detonante para la elaboración de planes de adecuación urbana que respondían no solo a las necesidades ya citadas, sino también al interés de las élites paceñas en consolidar su poder político, social y económico por medio del fortalecimiento de la ciudad de La Paz como centro de ese poder. Esto se traduciría por un lado en la elaboración del discurso liberal de modernidad y, por el otro, en la búsqueda de modernización del espacio urbano paceño. Tomando en cuenta este contexto, el presente análisis trata de dilucidar la forma en la que el discurso y las prácticas derivadas de la idea de "modernidad" y la construcción del espacio urbano a principios del siglo XX tuvieron un rol sobre la población indígena urbana en La Paz.

Al hablar de "modernidad" nos referiremos principalmente al discurso en torno a la concepción de la misma de fines del siglo XIX. Esta, no solo en Bolivia sino en el resto del continente desde fines del siglo XIX, se fundamentó no tanto en las ideas europeas de siglos anteriores sino en teorías como el positivismo, el darwinismo social, etc., además de la noción de la posibilidad de un desarrollo lineal y ruptura con el pasado. La lógica de ruptura, jerarquización, orden, posibilidad de desarrollo y modernización de todos los ámbitos acompañará al pensamiento de ensayistas, científicos y políticos. Citando a Latour, este enfoque les impedía pensarse en continuidad con los "premodernos" (Latour, 2008) por lo que los mecanismos que serán nombrados en adelante son solamente una pequeña parte de la trama de este afán. El entusiasmo causado por la mejora de la economía y el aumento de la población hacía pensar en la posibilidad de ese desarrollo planificado. La otra cara de la moneda fue que, si bien se planteaba la ruptura con el pasado, no territarias 36 
${ }^{1}$ El biologismo es una serie de teorias e interpretaciones científicas que la configuran como ideología para la cual el ser humano no es más que el resultado de su berencia y configuración biológica. El proceso de la configuración del biologismo se inició a raíz de la industrialización, urbanización y la proletarización en Europa. (Harms, 2011).

${ }^{2}$ La teoría de las nuevas materialidades se enfrenta a análisis en los que la cultura material es tomada solo desde el aspecto representativo y semiótico. Esta propuesta teórica se diferencia de la del materialismo bistórico fundamentalmente en que ve a la materia o los artefactos culturales como agentes de cultura, como actores, mientras que el materialismo clásico la ve como objetos que forman parte de los procesos de acumulación, sin acción propia. Elénfasis en las materialidades puede verse como contrapunto a la asignación de poder que se dio a las construcciones discursivas textuales que ponian a los espacios y artefactos en un segundo lugar o que los reducian a meros escenarios. Además de considerar a los artefactos culturales como actoresy parte de redes homólogas a las de la sociedad (Miller, p. 7) a través de este enfo-

\section{territarias 36}

se tocaron las estructuras sociales y económicas más importantes heredadas. De hecho, en la mayoría de los casos (como en el boliviano) este desarrollo descansó en el tributo y trabajo indígena. Solo el cambio del "gesto" sería permitido en la medida en la que gracias a ello se podía incorporar a población al aparato económico y, en este caso, urbano.

De forma más concreta, puede verse como este discurso se basó en tres pilares: el primer aspecto fue el discurso, desde las ciencias humanas, hasta el conocimiento urbano y arquitectónico se le planteó como un "proyecto civilizatorio" y de "progreso", poniendo sus postulados como eje fundamental para repensar a sus sociedades. La aspiración de desarrollo lineal de las sociedades mediante el avance técnico y las técnicas sociales de disciplina y de incorporación al mercado serán uno de los puntos centrales. A su vez, la concepción lineal de desarrollo de las sociedades llevó a la confluencia de distintas construcciones biologistas ${ }^{1}$, que justificaban la superioridad de algunas razas sobre otras basados en la cercanía o lejanía con el proyecto civilizatorio. Bajo este discurso, se asumió la "naturalización" de las relaciones y separaciones y la necesaria superioridad de la ciencia sobre otros saberes (Lander, 2005 , p. 19). El segundo aspecto fueron las prácticas emanadas de estos discursos de poder y conocimiento que se enfocaron en la reconfiguración misma de la población por medio de una serie de medidas jurídicas y de policía enfocadas a la "habilitación de la población”. La ruptura con cánones tradicionales será uno de los lemas de este proyecto. Dentro de las estrategias encaminadas a este diseño pueden verse las referidas a la higienización y control de la sociedad, además de todas las directivas de ornato público, uso de medios de trasporte, etc.

El tercer aspecto fue la construcción física del espacio urbano siguiendo los dos criterios anteriores. Esta construcción física será tomada como parte del proceso de construcción de sujetos urbanos partiendo de la premisa de que el plano material, y en este caso físico-espacial, será un actor más en la configuración de la sociedad urbana paceña. Esta visión se enmarca en el de las nuevas materialidades y la Teoría del Actor Red que plantea que tanto actores humanos como materiales están inmersos en redes que conforman lo social (Latour, 2008). En este sentido, Latour propone analizar los momentos en los que los objetos se hacen visibles, momentos de innovación, por ejemplo, o de "controversias" más que de conflictos. Es en esos momentos, según el autor, en los que se posibilitaría analizar a todos los actores por ser visibles. En el caso a analizar, siguiendo esta línea, la controversia e innovación estaría dada en la ampliación del espacio urbano. En ese momento el plano físico jugó un rol fundamental en la reconfiguración de la red de actores urbanos.

Además de estos factores, debe tomarse en cuenta las características duales de este proyecto. Por un lado la supuesta búsqueda de "modernidad" y, por el otro lado, la permanencia de patrones sociales 
heredados de la Colonia. Diversos estudios hablan de esta situación (Larson, 2002; Demelas, 2003; Ergueta Villamor, 2009; entre otros), desnudando en cada uno de ellos la existencia de un discurso de modernidad junto con la permanencia de cánones de segregación de la población indígena. Dentro de esta construcción puede verse, además, las representaciones materiales tanto del proyecto de modernidad como de los sujetos que se iban diseñando alrededor del mismo. El juego entre estos factores marcará la conformación de un espacio urbano que intentaba modelar a sus habitantes. De hecho, las ciudades pueden ser tomadas como los artefactos más grandes y complejos creados por la humanidad (Hillier, 2007, pp. 111), serán los espacios privilegiados para ver la forma en la que por un lado se fue construyendo el proyecto modernizador y, por el otro, la forma en la que este, por medio de los cambios del espacio físico, influyó en la conformación de identidades urbanas y en el discurso acerca de lo indígena o la conformación del "indígena permitido" dentro del discurso nacional. Dentro de las concepciones de la época, el urbanismo y la arquitectura eran pensados como disciplinas que rompían con patrones de espacialidad y de hábitat considerados como "pre-modernos". Puesto que el boom del urbanismo en Europa y el resto de América Latina ya había mostrados sus frutos, desde la realidad paceña se tomó a dichas disciplinas como corolarios para el proyecto. No obstante, y siendo uno de los factores fundamentales que dotan a la ciudad de La Paz con ca- racterísticas individuales frente a sus pares sudamericanos, esta etapa coincide con la del indigenismo y las primeras grietas ante el discurso de modernidad. En este sentido, y principalmente en la arquitectura, se iniciará un proceso de unión entre las escuelas contemporáneas de arquitectura con las de rescate y revalorización de la herencia indígena ${ }^{3}$.

A diferencia de lo ocurrido en la Colonia y parte del periodo Republicano, en los que las fronteras espaciales entre el mundo "blanco" y el indígena pretendían estar delimitadas de forma física y no solo simbólica, en esta etapa se imponen criterios de marginación distintos. La ciudad crece y tanto la movilidad como el uso del espacio público se convierten en uno de los factores determinantes de la misma. El uso del transporte motorizado será uno de esos factores materiales y, gracias al mismo, la expansión de la ciudad y la movilidad en la misma irá perfilando espacios que ya no podían ser controlados como en décadas anteriores, por lo que lo qué se pasará a regular serán las actitudes y costumbres urbanas. Por lo tanto la alteración de vestimenta, la higiene y criterios de urbanidad serán las nuevas fronteras que irán perfilando a los habitantes de ese espacio. Se pasa de la separación de los espacios a la homogenización aparente.

Las formas de adueñarse del espacio se irán modificando en una suerte multifactorial: el discurso de modernidad dará paso al proyecto de sociedad a ser diseñada, lo que se refleja en la construcción del nuevo espacio urbano. El lugar y rol que se pone en cuestionamiento la separación entre materia y espiritu o entre artefacto e individuo. Según esta propuesta, sería erróneo hablar incluso de una reconciliación entre el mundo material y social, ya que incluso esta división es una invención (Latour, 2008, p. 112). En este sentido, esta propuesta teórica propone un proceso circular en el que no pueden diferenciarse los sujetos per se de los objetos per se (Miller, p. 14), eliminando el dualismo entre objeto y sujeto. Para Latour esta circularidad es la que permitiría realizar un análisis distinto al dialéctico que entierra a los objetos viéndolos como cuestiones superadas (Latour, 2008, p. 243). La circularidad permite hacer visible las "largas cadenas de actores" (Latour, 2008, p. 249) que se vinculan $y$ actúan entre sí a manera de red. Esta red, a su vez, puede ser analizada a través del rastro de las relaciones de las "controversias" en las que los actores están inmersos y que dan pautas de las conexiones sociales (Latour, 2008, p. 42). Esto implica, además, ver a los actores en un proceso de movimiento. Movimiento que genera reasociación y reensamblado. De esta manera, se define a lo social ya no como lo relacionado solo a sujetos dentro $\Longrightarrow$

territarias 36 
$\Leftarrow$

de una sociedad, sino a todos sus actores en movimiento $y$ circularidad asumiendo que tanto los sujetos como los objetos forman parte de la misma red interactúan permanentemente. Gracias a estas interrelaciones, más que a la existencia de un entorno objetivo y otro subjetivo o actores humanos $y$ no bumanos, puede hablarse de la unión de ambas en lo colectivo (Latour, 1999, p. 30). Para analizar a estos actores Latour propone analizar tanto la continuidad como la discontinuidad de los modos de acción, los momentos en los que los objetos entran en relación con lo social e incorporar a los objetos dentro de los relatos.

Para realizar este tipo de narracionesse propone analizar las innovaciones y controversias, la "novedad", los accidentes, los archivosy las ficciones (pp. 119-121). Es decir, los “momentos" en los que los objetos se hagan presentes, ya que los mismos, en instantes en los que no interactuan con entes "sociales”, pasan a un segundo plano.

${ }^{3}$ Si bien en este artículo no se ahondará en este tema, consideramos que no puede dejarse de lado este aspecto que en décadas posteriores marcará la incorporación visual de lo indigena en la arquitectura. Basta $\stackrel{\text { ver }}{\Rightarrow}$

territarios 36 74 asignado a los cuerpos en la ciudad variaría en este periodo respondiendo al discurso, creando nuevas actitudes y prácticas. La lógica del desarrollo va exigiendo de los habitantes urbanos una reconfiguración de las formas de apropiación del espacio y de actuación en el mismo. Cada espacio público será objeto de regulación. Cada medio de transporte, cada estructura de salud y educación. Podría afirmarse que la ciudad en este periodo histórico actuó como un dispositivo (Foucault, 1991) de operatividad del proyecto civilizatorio de la modernidad en esta región. Así como en etapas anteriores se trató de estrategias de confinamiento, en esta etapa, y siguiendo siempre los cambios históricos y de conocimiento, los cuerpos serán reubicados. Ya no solo se tratará de su docilidad sino de su ingreso en el aparato productivo y el discurso nacional. Se trataría, de esta manera, de un dispositivo que mediante el control, la jerarquización y homogenización (aparente) de la población buscará la incorporación de los diversos actores al proyecto. De hecho, el rol asignado a los cuerpos dentro de los espacios urbanos irá variando a lo largo de la historia y según el contexto en el que se dio y respondiendo a los avances técnicos, como en el caso de la movilidad de los mismos (Sennett, 2007). Este hecho responde a la forma en la que los cuerpos fueron designados a lugares y roles específicos en esta etapa. El lugar que se permitirá ocupar y la regulación de tal uso serán fundamentales para comprender la forma en la que se va diseñando a los sujetos urbanos en este periodo. En etapas anteriores la arquitectura misma estuvo destinada a la asignación y control de los cuerpos en los espacios externos y sobre todo internos, como en el caso de la arquitectura militar (Schäffner, 1996), la de salud y la de confinamiento (Foucault, 2000).

\section{La ciudad de La Paz a los albores del siglo XX}

La presencia indígena en las ciudades andinas en general y bolivianas en particular marcó un tipo de modernidad que podría llamarse modernidad andina. Como dato estadístico, puede mencionarse que según el censo de 1900 elaborado a nivel nacional, la población total alcanzaba el 1.633.610 habitantes, de los cuales 445.616 se encontraban en el departamento de La Paz y 60.031 en la ciudad de La Paz. Ya para este censo se había sumado la población de "la Capital y la de área rural de los suburbios" (Dick, 2013, pp. 85), pasando a 115.000 en 1920 de los cuales el $70 \%$ era de ascendencia indígena (De Mesa, 1989, pp. 20). Como puede intuirse, la mayoría indígena de la población urbana puso un matiz indeleble en la organización y forma de vida en ese espacio. Si bien las ordenanzas municipales, herederas de la Colonia, y el discurso de división del espacio "blanco" y el indígena permanecieron, el día a día mostraba una realidad de convivencia y de heterogeneidad en el espacio público y privado ineludible.

Mientras que otras capitales latinoamericanas se enfrentaron a un proyecto 
de rediseño de sus ciudades capitales, en Bolivia, gracias al traslado de la sede de gobierno después de la Guerra Federal de 1898 , este proceso se trató más de la construcción de una ciudad que de su adecuación. En menos de sesenta años dicha ciudad pasó por etapas de diseño urbanístico y arquitectónico que en otras latitudes habían tomado más tiempo. La rapidez de este proceso puede observarse en las fuentes referidas a las cartografías urbanas de esta ciudad y a los proyectos urbanísticos, además del diseño mismo de la ciudad y sus operaciones que irán acompañados por un corpus de normativas y lineamientos de control urbano que se inscribirán en los mecanismos culturales de procedimientos de control, vigilancia, jerarquización y homogenización. Estas técnicas, a su vez, reflejan el contexto discursivo de modernidad en torno a la ciudad. La necesidad de la ciudad de La Paz de pensarse a sí misma como centro político dio lugar a un movimiento de modernización urbana sin precedentes que dio paso a una serie de readecuaciones en torno al uso y apropiación del espacio público, además de la inminente necesidad de expansión de la mancha urbana. Estos aspectos tenían el fin de consolidar una población urbana disciplinada, homogenizada e higienizada.

Como pueden verse en escritos de la época, las corrientes biologistas daban varios diagnósticos y supuestas soluciones a la problemática del "pueblo" boliviano. $\mathrm{Mu}$ chas de las costumbres y formas de apropiación del espacio urbano serán vistas desde las posturas higienistas como atentados a la urbanidad y factores de "degeneración racial", como es el caso del consumo de la hoja de coca (Carrasco, 1945). En este sentido, uno de sus principales intereses fue el de asignar un lugar y rol a la población indígena urbana. A falta de masas obreras que disciplinar, el mundo indígena fue visto como el foco para el accionar de las operaciones de modernización de la sociedad y el espacio urbano.

Desde mecanismos de control y operaciones culturales de apertura o cierre del espacio ${ }^{4}$ y de movimiento en el espacio urbano, la ciudad va ejerciendo un rol fundamental en el reordenamiento de códigos y prácticas culturales, económicas y sociales. El reordenamiento urbano en este periodo tuvo un papel importante sobre la forma en que se asumió "lo indígena", dando lugar a la incorporación pasiva de lo indígena en el espacio urbano y el discurso nacional ya que fueron el Estado y las élites los que pretendieron asignarle un lugar y un rol. Incluso dentro de los discursos indigenistas se idealizaba a las culturas precolombinas, mientras que los indígenas "contemporáneos" eran asumidos como "menores de edad" permanentes. A principios de siglo este aspecto era evidente en las políticas de integración de la población indígena en programas de educación. Es a partir de la Guerra del Chaco y los cambios que esta trajo que se comienza a poner en tela de juicio este aspecto. La lógica pasiva de incorporación será rota por el accionar público grupal de los indígenas desde el Primer Congreso Indígenal en 1945 y la Revolución Nacional de 1952 y, ya en los obras arquitectónicas como el Monoblock de la Universidad Mayor de San Andrés para apreciar esta fusión entre el funcionalismo y la linea escalonada encontrada en monumentos arqueológicos de Tiwanaku. Para ahondar en el tema pueden verse las obras físicas a manera de edificios y las escritas del arquitecto Emilio Villanueva (Villanueva E, 2014).

${ }^{4}$ Dentro del enfoque del análisis material de los espacios está el punto de vista operacional brindado por Wolfgang Schäffner, quien pone un especial énfasis en las formas de construcción de conocimiento espacial a través de acciones concretas. El autor pone a consideración el factor operativo de la arquitectura. Lo importante en su enfoque es ver qué operaciones modelan $y$ posibilitan interacciones, como en el caso de las operaciones de Apertura y Cierre (Schäffner, 2010).

territarias 36

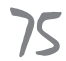


90, con las marchas indígenas en torno a la soberanía y autodeterminación. La irrupción de los delegados indígenas del Congreso en la Plaza Central fue una de las primeras apariciones de estos actores en el espacio público en grupo y no de forma individual. La prensa de la época, que antes del evento no le dio mucha importancia previa al hecho, reportó días más tarde sobre la participación de los líderes del Congreso, mostrando especial fascinación por la puesta en escena de los mismos. La llegada de 1.200 delegados al evento (El Diario, 1945) junto con la presencia de autoridades de gobierno fue uno de los primeros pasos para la participación política institucionalizada para los indígenas. No obstante, este hecho coincide con la utilización de la denominación de campesino para las comunidades ubicadas en el altiplano. Esta categoría sería utilizada en adelante y sobre todo en la época de la Revolución de 1952, significando su ingreso discursivo a la modernidad. La eliminación de la categoría "indio" y su cambio por "campesino" sería una nueva forma de homogeneización y mecanismo de acción sobre esta población, negando las formas tradicionales de tenencias de tierra y organización.

Sin embargo, y a pesar de que la memoria corta del movimiento indígena (Rivera Cusicanqui, 1986) se basa en estos hechos, la importancia de ver el periodo de inicios del siglo XX, el de la incorporación pasiva de lo indigena en el discurso nacional, se basa en que, a partir de él, se sentaron las bases de justificación de los indígenas como actores nacionales legítimos en el correlato histórico. Evidentemente la acción del movimiento indígena y sus reivindicaciones tuvieron un rol fundamental en el mismo, sin embargo, de parte de la administración urbana y sus procesos de construcción urbana jugaron procesos basados en el discurso de control e higiene y la instalación de prácticas de segregación física y discursiva a partir de criterios "racionales", es decir, legitimados desde el discurso de las ciencias, la modernidad y el progreso. Las políticas y operaciones emanadas de las medidas de modernización del espacio urbano paceño muestran cómo este dispositivo actuó en la implantación de parámetros y formas de convivencia.

El relacionamiento con el área rural a partir de la conformación de la ciudad como punto de modernidad y civilización, se estableció precisamente a partir de estos parámetros de convivencia y prácticas de urbanidad. A pesar de que pueden buscarse resistencias directas a este proceso, lo que va encontrarse, en general, son estrategias de adecuación a los nuevos parámetros. Ya desde el siglo XVIII la población indígena que vivía en áreas urbanas se fue desprendiendo de formas de organización, vestimenta y convivencia convirtiendo a la ciudad en una suerte de bisagra entre dos mundos. La estrategia de mimetización con la población mestiza les permitía por un lado cortar con el tributo y el servicio impago de las haciendas y, por el otro, incorporarse a un mercado de profesiones "nuevas" como la confección y diversos talleres. En términos espaciales, los alrede- 
dores de la ciudad de La Paz, especialmente los aledaños a la iglesia de San Francisco, serán los que alberguen a estos nuevos oficios que se irían constituyendo con escenarios privilegiados de ascenso social.

\section{Apertura y control del espacio urbano paceño}

Hasta fines del siglo XIX la ciudad de La Paz se encontraba jurídica y discursivamente dividida entre un centro "blanco" y el espacio ocupado por las parroquias de indígenas que se encontraban en los "barrios altos" (Saignes, 1985, pp. 288). En la monografía de la ciudad de La Paz de 1902 se señala la diferenciación de un espacio urbano y uno rural ubicado en los territorios de esas antiguas parroquias (Crespo, 1902, pp. 90).

A pesar de que la barrera entre ambos era casi imperceptible ya en siglo XIX, puesto que la población indígena que vivía dentro de la ciudad o en las parroquias fue absorbida paulatinamente (Barragán, 1990, pp. 82), pervivió una especie de $s e-$ paración simbólica por medio de una serie de operaciones tanto físicas como discursivas. Si bien la expansión de la ciudad a los barrios extramurales había sido continua incluso dando paso a pleitos de tierras, este hecho se daría de forma desordenada y respondiendo más a criterios de necesidad de apropiación de tierras que a un criterio urbano. Será a fines del XIX e inicios del $\mathrm{XX}$ que se piensa en la incorporación de dichos espacios desde la planificación y diseño urbano.
La división del espacio urbano paceño no solo se mostraba en la forma de tenencia de tierra o de la población de los barrios, sino también en una barrera físico-simbólica a manera de fortificación. La primera fortificación de la ciudad fue ordenada raíz de los levantamientos indígenas que se dieron en la región. Gracias a ella se dejaba fuera de los límites de la ciudad a los “arrabales" y poblaciones de indios (Del Valle de Siles, 1980, pp. 41), este límite implicaba un mecanismo de control de movimiento en el interior de la ciudad por medio de la imposición de puertas en los puentes que unían al centro con el resto del territorio (Gisbert, 1977, pp. 58). En el plano de la ciudad de La Paz elaborado por Florentino Olivares en 1888 (figura 1), y en su representación de este cerco, se muestran dos espacios claramente diferenciados: el espacio blanco heredero de las concepciones de orden y de planificación hispana y, por el otro, el indígena, como representación de un espacio abierto, indefinido, en el que solo las iglesias son representadas como puntos de ordenamiento. Esta representación mantuvo un hilo conductor con los planos anteriores (1796, 1859, 1876) en los que los sectores extramuros eran representados con línea puntuada, línea difusa, etc. y con ausencia de nombre o delimitación certera de calles.

La idea de la ciudad de La Paz como acechada por el mundo indígena se conservaría, en adelante, por una serie de relatos en los que la "peligrosidad" indígena sería la principal razón para la elaboración de territarias 36

77 
Figura 1: Florentino Olivares, El cerco de La Paz 1781. Elaborado en 1888.

Óleo sobre tela, $142 \times 186 \mathrm{~cm}$

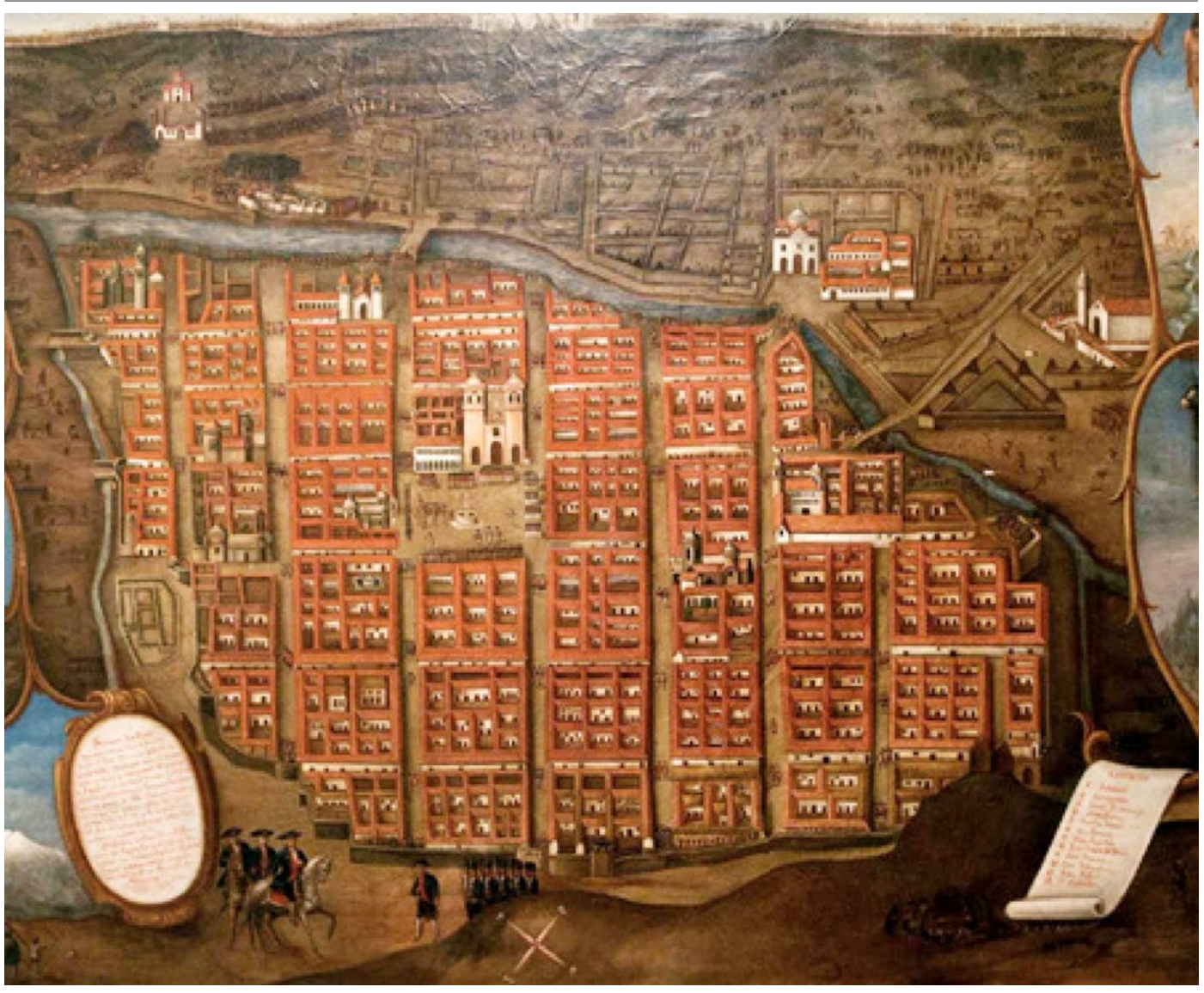

Fuente: Museo Casa de Murillo La Paz, Bolivia

mecanismos de defensa física del espacio urbano "blanco". El primer plano de la ciudad que incluía los dos márgenes del río fue el elaborado por la Comisión Topográfica en 1902 (Gisbert, 1997, pp. 62) para la Monografía de La Paz de 1903 (figura $2)$. Hasta ese momento, los dos espacios eran claramente diferenciados, a pesar de que en las últimas décadas del siglo XIX la delimitación de las manzanas de los barrios inmediatos a la ciudad blanca iban consolidándose en dichas representaciones (Bedoya, 1998). Sin embargo, la particularidad de este mapa radica en que hizo parte del esfuerzo de redefinir ese espacio y fue parte del discurso de inclusión de los territorios 


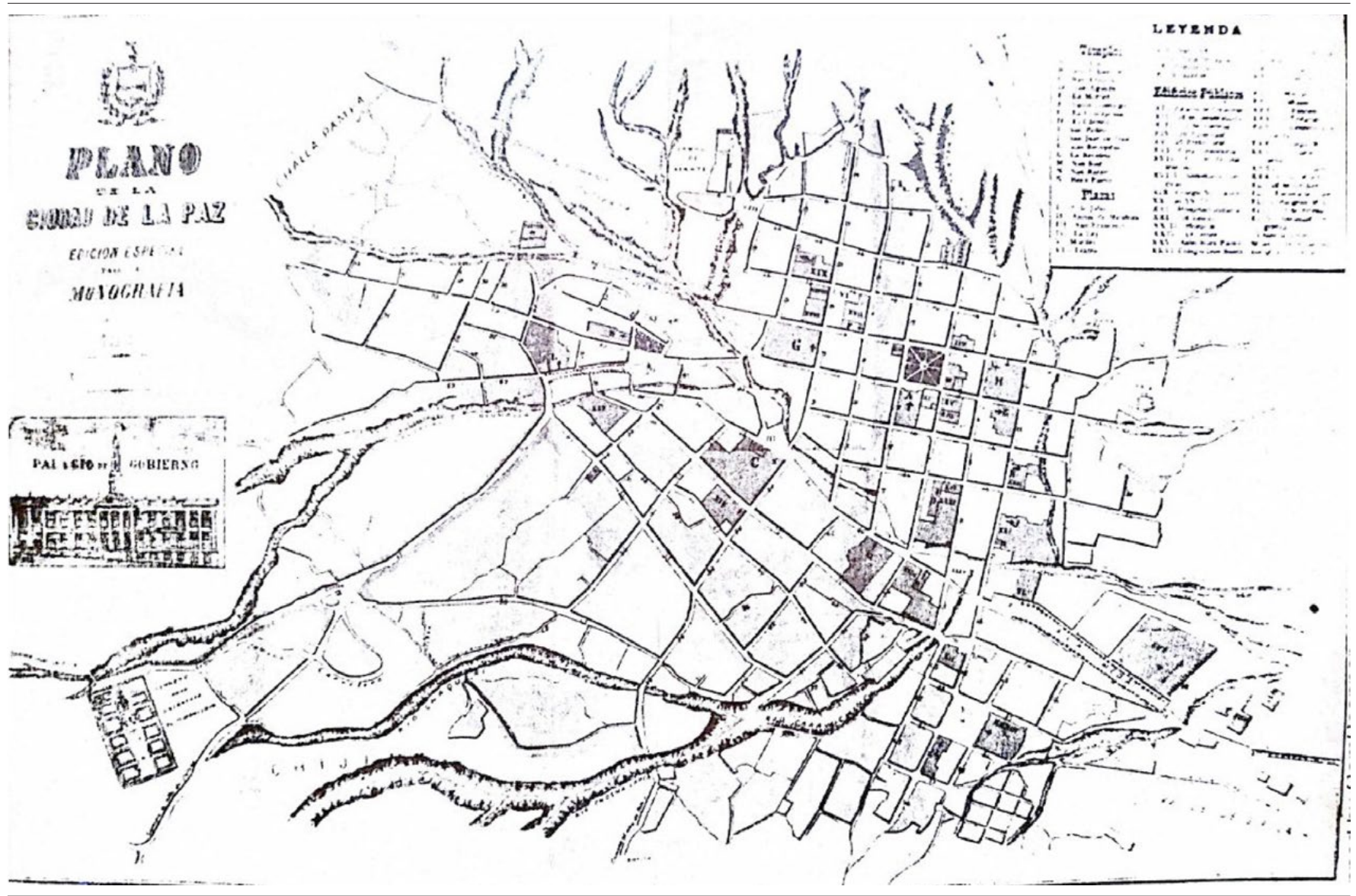

Fuente: Edición especial para la Monografía de La Paz. (Bedoya, 1998).

anteriormente conocidos como indígenas. El resto de las laderas continuaron siendo representadas de forma difusa.

Ante este panorama, la ciudad emprende la empresa de la operación de apertura. Ello implicó la eliminación físico-simbólica de la diferenciación entre los dos espacios. El espacio blanco y los barrios indígenas serían, en adelante, incorporados al relato urbanístico paceño. Esta inclusión siguió a su vez una serie de mecanismos: el primero, la unión del centro con el resto del territorio paceño. Hasta ese momento, los dos espacios se encontraban conectados por puentes sobre el río (Crespo, 1902). La eliminación de los mismos para la construcción de vías de comunicación y entubado será el primer paso para la territarias 36 
apertura de ese espacio. Posteriormente se construyeron barrios inspirados en el estilo europeo y se comenzó a usar medios de transporte de fuerza motor para conectar las distintas zonas.

La eliminación física de la barrera topológica entre la ciudad blanca y la indígena se dio paulatinamente desde 1914 gracias a la planificación y entubamiento del río Choqueyapu que separaba ambos espacios (De Mesa, 1989). Con esta acción, a cargo del arquitecto Emilio Villanueva, se iniciaba además el proceso de planificación urbana y arquitectónica especializada que incorporaba criterios de urbanismo europeo como el de los paseos. Siguiendo esta planificación, y sobre el río, la vía que partía desde el norte de la ciudad se uniría al paseo de La Alameda, conocido actualmente como El Prado. Este espacio fue uno de los primeros en ser construidos a manera de punto de fuga del centro, convirtiéndose más adelante en la principal vía de comunicación con los barrios nuevos. En él, además, los actores se mostrarían en una suerte de espacio de autorepresentación.

Estos cambios fueron paulatinos y trajeron consigo el accionar sobre la población para adaptarse a los mismos. Como primer punto, se planteó la especialización de los espacios urbanos, es decir la asignación de roles y normativas a cada uno de los ellos. Pueden citarse varios ejemplos: uno de ellos el de las fiestas, a las que se había tratado de prohibir desde mediados del siglo XIX por ser consideradas como costumbres bárbaras (Barragán, 1990, pp. 66), argumentación que cambia a prin- cipios del siglo $\mathrm{XX}$, cuando comienza a pensarse en su regulación como práctica urbana alejándose del contenido ritual. En este sentido, los debates ya no serían en torno al nivel de civilización o paganismo, sino más bien sobre el uso o mal uso del espacio público en tanto espacio ordenado e higienizado.

Otro ejemplo será el de los mercados. Atrás quedaba el mercado heterogéneo y desordenado, o las calles estrechas y llenas de olores. La premisa fue el control de estos espacios en pro de la idea de higiene y salubridad. Tal fue el caso de la ordenanza municipal de 1906 referida al mercado que se encontraba en el atrio de la iglesia de San Francisco, en ella las chifleras eran expulsadas de ese espacio y con estas vendedoras de plantas, el conocimiento sobre hierbas y medicina indígena. Este caso es paradigmático, ya que hasta aquel entonces, la iglesia y atrio de San Francisco eran el centro de la ciudad extramuros. A pesar de que antes de la apertura del espacio urbano "blanco" varias construcciones en los barrios indígenas ya se encontraban en manos de las élites, la regulación del espacio público sería la que realmente marque la apropiación del mismo bajo las premisas de modernidad. Las ideas de salubridad, orden e higiene serían las que jugaran en la construcción del espacio del otro. A partir de él, las diferencias ya no se argumentarían a partir de jerarquías raciales, sino de actitudes y de gestos. La modernidad llamaba a la población urbana a adoptar los preceptos de urbanidad y salubridad para ser parte de ese proyecto civilizatorio. En 
este sentido, se ensayaron diversas medidas de expulsión de personajes como los mendigos, buhoneros, chifleras y otros de los escenarios centrales de la vida urbana y de la infraestructura recientemente creada, sin que estos desaparecieran realmente. En el caso mencionado, y dejando la posibilidad de un futuro estudio sobre la forma en la que se consolidó un espacio alterno para su actividad, las chifleras se alejan del mercado de San Francisco a tan solo unas cuadras en lo que actualmente se conoce como la calle de las brujas. Este ejemplo es paradigmático en el sentido que marca una de las estrategias principales de los sectores populares ante las medidas de control. Siendo un terreno que aún debe investigarse, nos arriesgamos a afirmar que la estrategia de alejarse de los espacios de control hacia barrios o calles periféricos logró que tanto prácticas como imaginarios considerados como "indeseables" para las normativas municipales lograsen sobrevivir hasta nuestros días, otorgando incluso matices particulares a esta ciudad.

Dentro del proyecto urbano, se ve al otro a partir de la cercanía o distanciamiento de la concepción de "modernidad" y que se evidenciaba mediante su comportamiento y puesta en escena en el espacio público. Aunque aparentemente la apertura del espacio urbano y los mecanismos que ello supuso acababan con la ciudad indígena, los mecanismos de representación y control pasaron a configurar espacios diferenciados a partir de las formas de apropiación del espacio. Una vez que la ciudad se abre, las clases altas paceñas pasan a ocupar terrenos lejos del antiguo centro histórico. Es el momento de la arquitectura estilo chalet (Sopocachi), y el diseño de la ciudad jardin (Miraflores) inspirada en el urbanismo francés. Las formas de las viviendas y la arquitectura moderna marcarían una nueva frontera, dejando en manos de las clases populares los antiguos espacios señoriales. Imponiéndose la frontera entre lo moderno y lo colonial o premoderno. La apertura del espacio paceño, de esta manera, no significó la incorporación de los sujetos que habitaban ese espacio adquirido. Al contrario, se instauraron nuevas formas de segregación y jerarquía. Como se adelantaba, el primero de esos factores fue el de la regulación y especialización de los espacios, la regulación de la puesta en escena de los cuerpos en el espacio urbano, y la limitación del uso de medios de trasporte.

En cuanto a la regulación de este espacio y la puesta en escena de los cuerpos, puede ponerse como ejemplo al reglamento sobre indumentaria indígena de 1904 (Pinilla, 1905), donde se ordena a los indígenas adoptar el traje de los criollos, por considerar que el traje tradicional había sido un factor de su atraso. En el mismo se señala que la única diferencia física entre ellos y los mestizos sería de esta manera "superada" dando paso a la superación de sus condiciones. En esta reglamentación no solo se aconsejaba su utilización, sino que se pasa a prohibir a los sastres su confección. La justificación de la misma era clara: respondía al discurso higienista de la época y la necesidad de territarias 36 
reglas de higiene además de la necesidad de "elevar el nivel moral y material del pueblo". En dicha normativa, se señalaba que la presencia de los indígenas en espacios públicos o fiestas patronales portando sus vestimentas (o lo que se asumía como sus vestimentas) sería multado obligándoles además a abandonar el lugar. Se identifica a la vestimenta como un criterio de evolución social, viéndola como un factor de “adelanto" o "retraso" (Paredes, 1911, pp. 11). La idea de que la alteración de la vestimenta era un factor de civilización sobre los indígenas responde a su vez a los discursos biologistas del siglo XIX, a pesar de que lo que en aquella época se asumía como vestimenta indígena ya había pasado por un largo proceso de transformación desde la llegada de los españoles. Además de ello, varios testimonios dan cuenta de que especialmente la vestimenta masculina era prácticamente indiferenciable de la de los mestizos, mientras que la femenina se iba adaptando también adoptando las primeras versiones de lo que se conoce actualmente como polleras. No obstante, el deseo de una población homogénea, en la que tanto la vestimenta como las actitudes estuvieran acordes a la "los nuevos tiempos" se incrementa con la entrada de los trasportes masivos. Lo llamativo del caso, es que el cambio de vestimenta se planteaba con la homogenización con la población mestiza, jamás con la de las capas "blancas" que adaptaban su vestimenta a la moda europea.

La incorporación de las técnicas de tersitarias 36 82 será el tercer factor de configuración de esta época. De hecho, la construcción del ferrocarril Arica-La Paz fue uno de los aspectos fundamentales de la construcción de infraestructura urbana (Sanjinés, 1948, pp. 47). La idea de "progreso" y el movimiento de capital y tecnología a partir de este proyecto llevó a la construcción de infraestructura urbana como la Gran Avenida de La Paz en 1912, y el sistema de tranvías y avenidas aún en perjuicio de los hacendados urbanos. El tranvía movido por fuerza motorizada entra a La Paz en 1909, con líneas que conectaban al centro con Sopocachi, Miraflores y el Cementerio General (Lázaro, 2009).

La implementación del trasporte masivo permite la ruptura de la lógica espaciotiempo que había mantenido hasta ese momento un espacio urbano compacto. La posibilidad de trasladarse en poco tiempo de un barrio a otro posibilitaba a las clases altas "alejarse" del centro y de los barrios populares, sin que ello se tradujera en renunciar a las comodidades del trabajo doméstico que éstas les brindaban. Es el caso de las culinarias (Wadsworth, 1989), mujeres de origen indígena o mestizo que se dedicaban al servicio de elaboración de comida a domicilio de las clases altas. El tranvía les posibilitaba el transporte rápido de un domicilio a otro, no obstante, una normativa de 1935 les prohibía el ingreso a los tranvías con las tradicionales canastas que éstas llevaban. La resistencia a esta medida, y las consecuentes protestas que encabezaron hasta que se derogó, muestra ya el cambio de la participación pasiva 
a la activa en el espacio público. Además del significado asignado a los trasportes masivos, éstos no solo eran considerados como objetos que formaban parte del entorno urbano, sino como símbolos de la posibilidad de apropiamiento del espacio urbano y la ruptura de la fragmentación simbólica del mismo.

\section{El discurso de la diferencia en el entorno urbano}

El carácter heterogéneo de las ciudades permite ver su constitución por medio de varios enfoques, unas veces desde lo económico y otras veces desde lo social, el uso del espacio público, el acceso a recursos, etc. En este ensayo pretendimos presentar otro criterio: el de la construcción imaginaria de fronteras por medio de operaciones urbanas y las prácticas de apropiación del espacio urbano unidos a la apropiación de artefactos y medios en este entorno. La unión de estos tres factores en el periodo analizado permite observar la forma en la que se fueron creando criterios de diferenciación a raíz del discurso de modernidad y progreso. Diferenciaciones que, si bien continuaron con la tónica colonial y republicana de la construcción de $l$ otro, tuvieron la particularidad de unir los discursos de la ciencia y el conocimiento con los de salubridad, orden y control en el entorno urbano, planteando a las ciudades como paradigma de materialización de este discurso. El crecimiento vertiginoso de las ciudades, con todo el movimiento político, cultural y económico a los que se asistió en aquel entonces, correspondería con la pervivencia de la idea de ciudad como sinónimo de modernidad.

Las barreras urbanas impuestas como códigos de diferenciación en la sociedad boliviana de la época permiten observar la segregación a partir de la argumentación higienista y de urbanidad. La construcción física de barrios en los que se pudiera vivir un nuevo sueño de orden por medio de la planificación urbana y el uso de medios de trasporte implicó un nuevo juego en el que los roles fueron asignados a partir de la proximidad o lejanía con el proyecto de modernidad urbana. El criterio de urbanidad, sumado al de la ciudadanía y al del discurso nacional fueron los mejores mecanismos de la introyección de dichos criterios. Mientras que en épocas anteriores se puede hablar de discernimientos principalmente represivos e impuestos, lo particular de este periodo es que dichos mecanismos, por medio de todo un aparato de control y homogeneización, actuaron no solo como criterios impuestos como represión, sino como posibilidad de adhesión y de inclusión en el relato de progreso y de nación. En este mismo sentido, puede entenderse que mecanismos como el de la educación o la inclusión al servicio militar fuesen peticiones que a la larga respondían a la posibilidad de adquirir el status de ciudadano, y por lo tanto pasar a ser parte del proyecto. Si bien esta posibilidad fue tomada, el diseño de nuevos mecanismos de exclusión como la codificación de reglas de usos de los espacio y medios, iban generando nuevas tipos de diferencias, ya no territarias 36

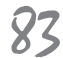


raciales (aunque tenían mucho que ver con ellas) sino de costumbres y procedimientos. La diferencia se iría argumentando cada vez menos desde los discursos de raza, pasando a otros más sutiles. Es por ello que puede entenderse mejor el rol central de la vestimenta, el uso de los espacios como los mercados o de los medios de transporte como los tranvías. Estos factores que juegan entre los artefactos, los espacios y los procedimientos culturales otorgan una radiografía en la que pueden adivinarse los nuevos criterios de diferenciación que afectan a este entorno hasta el día de hoy.

Si bien los conflictos en aquella época no pueden compararse con los actuales, sí se puede establecer unos criterios de influencia en la situación actual en la que el espacio en el que se reside en la ciudad marca de forma indeleble el origen, nivel de educación, ingreso económico, etc. Estos criterios son similares en muchas ciudades latinoamericanas, si bien las ciudades crecieron, se fue estableciendo paulatinamente la diferencia entre los barrios planificados y los de invasión (por clasificarlos de alguna manera arbitraria). Los primeros, al igual que los de principios de siglo XX, se identifican con el proyecto de expansión urbana y de incorporación en el relato de "ciudad moderna" que aún se utiliza, mientras que los segundos se van localizando fuera de este discurso, con todo lo que ello implica. Las imágenes de barrios privados y cercados son el extremo de esta diferenciación. El cierre del espacio urbano convirtiéndolo en un entorno mixto al ser público para los habitantes de dichos barrios pero privado y vetado para el resto. En el presente, el discurso de la seguridad puede verse como un heredero de los discursos salubristas. La enfermedad en este sentido dio paso a la criminalidad. La categorización de zonas enteras como "peligrosas" es la consecuencia más conocida de esta construcción. Cabría preguntarse nuevamente cuáles con las consecuencias de esa nueva fragmentación del espacio urbano.

\section{Referencias}

Barragán, R. (1990). Espacio Urbano y Dinámica étnica. La Paz en el siglo XIX. La Paz: HISBOL.

Bedoya Ballivian, M. (Comp.) (1998). Planos de la ciudad de Nuestra Señora de La Paz a partir del siglo XVI. La Paz: Honorable Gobierno Municipal de La Paz.

Carrasco, B. (1945). La engenesia en el ambiente boliviano. Oruro: Sociedad de Eugenesia de Oruro.

Crespo, L. (1902). Monografía de la ciudad de La Paz. La Paz: Ed. Sociedad Geográfica de La Paz.

Dick, G. (2013). Bolivia de Ayer. De un siglo a otro: del XIX al XX. La Paz: Imprenta Editorial Túpac Katari.

Rivera Cusicanqui, S. (1986). Oprimidos pero no vencidos. Luchas del campesinado aymara y qhechwa de Bolivia, 1900-1980. Ginebra: UNRISD.

Del Valle De Siles, M. E. (1980). Testimonios del cerco de La Paz. El campo contra la ciudad 1781. La Paz: Biblioteca Popular Boliviana de "Última Hora". 
Demelas, M. (2003). La invención Politica. Lima: Instituto de Estudios Peruanos.

De Mesa, J. (Coord.) (1989). 100 años de arquitectura paceña 1870-1970. La Paz: Colegio de Arquitectos de La Paz.

Con grandiosa sencillez se inauguró ayer el primer congreso de indígenas (11 de mayo de 1945). El Diario, p. 20.

Ergueta Villamor, A. (2009). El Darwinismo en Iberoamérica. Bolivia y México. Madrid: CSIC.

Foucault, M. (1991). Saber y Verdad. Madrid: Ediciones la Piqueta.

Foucault, M. (2000). Vigilar y Castigar. Nacimiento de la prisión. México DF: Siglo XXI.

Gisbert, T. (1977). Aspecto histórico-cultural. En G. Medeiros Anaya (Ed./ Comp/Org), Casco urbano central de La Paz. Tomo 4 CUC (pp. 56-72). La Paz: Honorable Alcaldía Municipal de La Paz \& Centro de estudios y proyectos nueva visión.

Harms, I. (2011). Biologismus. Zur Theorie und Praxis einer wirkmaechtigen Ideologie. Oldenburg: Verlag derCarl von Ossietsky Universitat.

Hillier, B. (2007). The Space is the Machine: A Configurational theory of architecture. Londres: Space Syntax.

Lander, E. (2005). Ciencias sociales saberes coloniales y eurocéntricos. En E. Lander (Comp.), La colonialidad del saber: eurocentrismo y ciencias sociales. Perspectivas latinoamericanas (pp. 1141). Buenos Aires: Consejo Latinoamericano de Ciencias Sociales (CLACSO).
Larson, B. (2002). Indígenas, élites y Estado en la formación de las Repúblicas Andinas. Lima: Instituto de Estudios Peruanos.

Latour, B. (1999). Die Hoffnung der Pandora. Frankfurt am Main: Suhrkamp taschenbuch wissenschaft.

Latour, B. (2008). Re ensamblar lo social. Una introducción a la teoría del actorred. Buenos Aires: Ed. Manantial.

Latour, B. (2008). Wir sind nie modern gewesen. Frankfurt am Main: Suhrkamp taschenbuch wissenschaft.

Lázaro, G. (2009). La energía eléctrica en la modernidad paceña. Represas de Milluni y Zongo, los tranvías y los inicios del teléfono. (1880-1950). La Paz: Consejo Municipal de La Paz.

Pinilla, M. (1904-1906). Memoria del Concejo Municipal del Departamento de La Paz.

Paredes, R. (1911). Altiplanicie paceña. El babitante y la población. La Paz: Boletín de la Sociedad Geográfica de La Paz. Año IX. Primer Semestre de 1911.

Saignes, T. (1985). Los Andes Orientales: Historia de un olvido. Cochabamba: IFEA \& CERES.

Sanjinés, A. (1948). La Paz. Sintesis histórica de la vida de la ciudad 1548-1948. La Paz: Imprentas Asociadas.

Sennett, R. (2007). Carne y piedra: el cuerpo y la ciudad en la civilización occidental. Buenos Aires: Alianza Editorial.

Schaffner, W. (1996). Schauplatz der Schauplatz der Topographie. Zur Repräsentation von Landschaft und Körper in der Niederlanden (15501650). En territarias 36

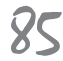




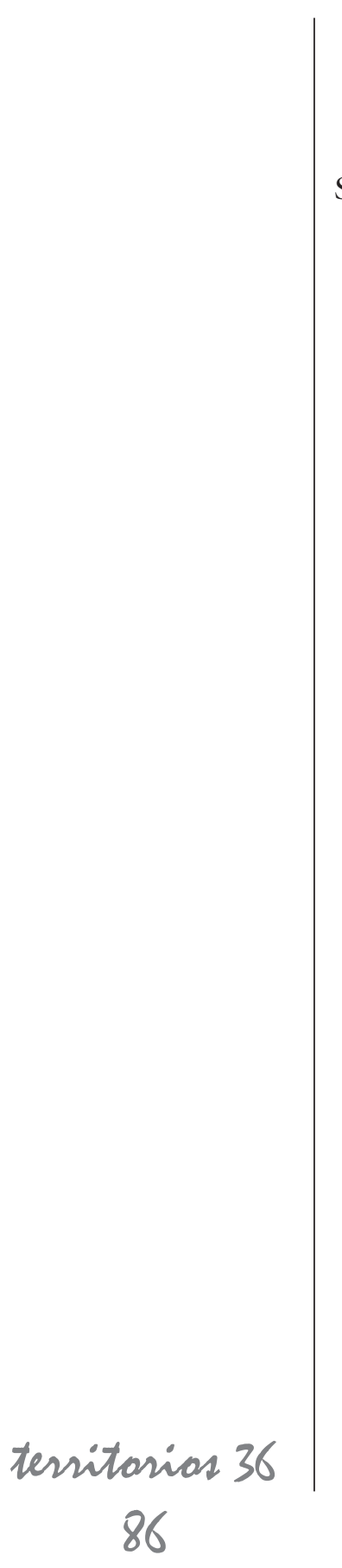

J.D. Müller (Hsrg.), "Auffübrung” und "Schrift" in Mittelalter und Früher Nenzeit (pp. 596-616). Weimar: J. B. Metzler.

Schaffner, W. (2010). Elemente architektonischer Medien. En L. Engell \& B. Siegert (Hrsg), Zeitschrift für Medien und kulturforschung. Schwerpunkt Kulturtechnik. 1/10 (pp. 137-150). Frankfurt: Ed. Felix Meiner Verlag. Wadsworth, A. C. \& Dibbits, I. (1989). Agitadoras del buen gusto. Historia del sindicato de las culinarias (1935-1958). La Paz: Tahipamu \& HISBOL. 